\title{
COGNITIVE
} SCIENCE

\section{The evolution of cognition-a hypothesis}

\author{
Holk Cruse* \\ Department of Biological Cybernetics, Faculty of Biology, University of Bielefeld, \\ Postfach 100131, D-33501 Bielefeld, Germany
}

Received 11 June 2001; received in revised form 13 February 2002; accepted 9 August 2002

\begin{abstract}
Behavior may be controlled by reactive systems. In a reactive system the motor output is exclusively driven by actual sensory input. An alternative solution to control behavior is given by "cognitive" systems capable of planning ahead. To this end the system has to be equipped with some kind of internal world model. A sensible basis of an internal world model might be a model of the system's own body. I show that a reactive system with the ability to control a body of complex geometry requires only a slight reorganization to form a cognitive system. This implies that the assumption that the evolution of cognitive properties requires the introduction of new, additional modules, namely internal world models, is not justified. Rather, these modules may already have existed before the system obtained cognitive properties. Furthermore, I discuss whether the occurrence of such world models may lead to systems having internal perspective.
\end{abstract}

(C) 2002 Cognitive Science Society, Inc. All rights reserved.

Keywords: Reactive system; Cognitive system; Deliberation; Internal world model; Recurrent networks; Internal perspective; Subjective experience; Consciousness

\section{Introduction}

There is a great variety of definitions of cognition, ranging from the traditional view that only humans-in contrast to lower animals or machines-are cognitive systems (review Atkinson, Thomas, \& Cleeremans, 2000) to the other extreme that "life is cognition" (Maturana \& Varela, 1992). In this article I would like to use a more practical definition and will show a concrete example of a system fulfilling the prerequisites required by that definition. To approach

* Tel.: +49-521-106-5533; fax: +49-521-106-6329.

E-mail address: holk.cruse@uni-bielefeld.de (H. Cruse). 
questions concerning cognition I would like to draw a distinction between reactive systems and cognitive systems, but will finally argue that this distinction is not a logically exclusive one. Being based on a holistic system the solution proposed here provides a possible explanation for the somewhat counterintuitive observation that some cortical neurons seem to belong to both the motor system and the perceptual system (e.g., mirror neurons: Gallese, 2000; Rizzolatti \& Arbib, 1998; bimodal neurons: Sakata, Taira, Kusunoki, Murata, \& Tanaka, 1997; decision neurons: Kast, 2001).

When investigating questions of how to control behavior, there are two types of problems. The first is concerned with the performance of a specific behavior (e.g., navigation) or of a specific function (e.g., pattern recognition) by the brain. Questions of this type are intensively investigated in psychological and biological research. The second problem, however, is studied much less frequently. This is concerned with the decision of which behavior to perform next, i.e., the problem of selection of action. In a realistic situation, this does not concern a decision between few, e.g., two, alternatives. Instead there is always a high number of possibilities. Such "redundant" tasks occur, for example, in pattern recognition (Ullman, 1995), speech production and speech recognition (Uszkoreit, 2000), or in motor control (Cruse, Brüwer, \& Dean, 1993). In all these cases, the decisions have to be made in situations characterized by a high number of extra degrees of freedom (DoF). The property of being able to cope with such redundant situations is often called autonomy. Experimental investigation of behavior usually concentrates on the first type of questions, because too much autonomy of the system studied makes the evaluation of the data intractable and should accordingly be avoided (except for early studies in ethology, e.g., Tinbergen, 1951). Consequently, a very important, if not the most important, property of a brain is excluded from experimental studies, namely its property to decide what to do next in situations characterized by a large number of DoFs (see also Edelman \& Tononi, 2000).

\section{Reactive systems}

The application of the research strategy to study only situations characterized by a small number of DoFs lead to a lack of knowledge that became most obvious when, in the field of robotics, autonomous robots were to be constructed. How should the robot decide what to do next? As not enough detailed results from behavioral biology had been known, the first approaches used solutions taken from traditional artificial intelligence inspired by insights gained from introspective methods used in philosophy and psychology. This led to the proposal that an internal, usually symbol-based, world model should be used to make these decisions. However, this approach turned out to lead to very slow and often inflexible solutions. Therefore, as an alternative, the behavior-based approach has been put forward (Brooks, 1991a, 1991b). It is inspired by biology and experimental psychology, and is based on strictly sensorily driven control of behavior, i.e., on reactive principles (e.g., Dean, Ritter, \& Cruse, 2000). Actually there are many examples which show that this approach can lead to the construction of systems with a considerable degree of autonomy (see the conference series on Artificial Life and on Simulation of Adaptive Behavior, among them the work of Beer, Chiel, Quinn, \& Ritzmann, 1998; Dörner, 1999; Maes, 1991; Pollack, 1991). 
As an example, Walknet, a system controlling six-legged insect-like walking shall be briefly explained. Walknet (Cruse, Kindermann, Schumm, Dean, \& Schmitz, 1998) is based on biological data and parts of it have been applied to different robots. It could be shown that this system which has to deal with 18 DoFs, and which is based on a group of modules consisting of simple artificial neural nets, has the following properties. The system can control walking in curves and on irregular substrate, it can cope with obstacles of different types, and it is tolerant with respect to sudden changes of body geometry. It also shows emergent, i.e., not explicitly implemented properties, such as different insect-like gaits or autonomous recovery from a fall. Like other similar approaches (Brooks, 1997), this system is characterized by a decentralized architecture, using restricted cooperation of different modules. Computation is extremely simplified by taking into account the loop through the world instead of using explicit computation, a property often characterized by the terms embodiment and situatedness. There are also "internal states" which means that a given sensory input can be responded to in different ways depending on the actual form of that internal or "motivational" state. These states described as swing state or stance state are determined by competitive modules which operate on a longer time scale than lower level modules and provide these lower level modules with some kind of limited protection against sensory input. As these states themselves are sensory driven, the whole system may still be termed a reactive system, however, in a broader sense (see also Sloman, 2000).

Furthermore, such reactive systems may be equipped with simple learning mechanisms like Hebbian learning or reinforcement procedures (e.g., Pfeifer, 1995; Parisi \& Cecconi, 1995; Tani \& Nolfi, 1999). These and other results show that the use of a behavior-based approach, i.e., application of reactive structures, can lead to adaptive controllers which can handle tasks characterized by a considerable number of DoFs (18 in the above mentioned case), and which therefore show a considerable degree of autonomy.

\section{Cognitive systems}

It is definitely a sensible research strategy to attempt to solve as many problems as possible by application of reactive structures. However, it appears not to be sensible to principally exclude the option that additional, non-reactive structures might be necessary in order to establish properties which may be called thinking, planning ahead or the like. According to the proposal of McFarland and Bösser (1993) and Lanz and McFarland (2000), in the present article, a system which has the ability to plan ahead will be called cognitive. Thus, systems with these properties can be distinguished from those described so far as reactive ones. It is a sensible assumption that for such a cognitive system some representation of the properties of the world, or, in other words, some kind of internal world model is required. Two types of representations will be distinguished here. The first type can be described as a "passive" representation, a static or lexicon-like memory, a look-up table. This type of representation is sometimes called a context-dependent world model, because, when using this representation, a given behavior can only be elicited in a given context and by a given stimulus situation. In a perceptron-like manner, a given stimulus triggers a specific response. Therefore, a system using such a passive world model can still be described as a reactive system. 
The second type may be termed a "dynamic" representation, or a "manipulable" world model. The content of this type of memory could be used to "play around with" in order to test different and new combinations of stored representational elements with the goal to find a, potentially new, solution to a given problem. In other words, such a world model allows to manipulate its content deliberately; the representation is "context-free." In this case the knowledge system should represent the mechanism underlying the content to be stored. Examples for the first type are associative memories, or lexicon-like AI systems. Manipulation of information, the second type, is usually performed by symbol-based AI systems (e.g., Aleksander, 2000; Nilsson, 1998).

In the following I would like to concentrate on the second type of world models. How could such a world model be constructed? In order to simplify this task, we may first ask for a possible basis, a primitive of such an internal model. What might represent such a primitive? The most important part of the world, and also the nearest part of the outer world, seen from a "brain's view," is the own body. This leads to the speculation that a basis of such a world model might be formed by a model of the own body (Cruse, 1999). Stating that the body changes much less than the external world does, Damasio (1999) argues along similar lines.

The necessity to assume the existence of an internal body model can also be made plausible by a completely different consideration. For this, we have to distinguish between two different situations occurring when a body with many DoFs is to be controlled: (i) The DoFs may be arranged in parallel. An extreme example is given by a milliped. Although each leg has only a small number of joints (for example, 3) the whole system is endowed with a high number of DoFs. However, for this case, Walknet, the above mentioned reactive system for the control of a hexapod could easily be expanded to control a milliped body. In other words, a reactive system would be sufficient. (ii) A more difficult situation occurs when the DoFs are arranged in serial order as in a multijointed human arm or an elephant trunk. For this case two solutions are discussed: (a) the number of DoFs could be reduced by fixed synergies (e.g., two neighboring joints may be controlled by a common control signal, Bernstein, 1967) or by the introduction of cost functions (e.g., the solution which requires minimum energy is selected). Such cost functions may vary with the task. Being fast but not flexible, these solutions are still of a reactive type. Their advantage is to simplify the problem and to permit fast solutions for known tasks. However, they have the drawback of lacking the possibility to exploit all geometrically possible configurations in order to find a solution for a new task. To make this possible, i.e., to be able to select in an ad hoc manner one out of all geometrically possible configurations, some kind of representation of all these possibilities - in other words, a representation of mechanisms underlying the system - appears to be necessary. Therefore, if the body to be controlled has a sufficiently complex geometry, an internal representation of the body geometry is needed or at least would be helpful. For example, Morasso, Baratto, Capra, and Spada (1999) convincingly argued that a body model is already necessary for the control of such a seemingly simple task as posture control in humans. Based on studies of phantom sensations, Melzack (1990) has postulated the existence of a neuronal representation of the geometry of the body and has introduced the term "neuromatrix" for this hypothetical network.

Internal world models are not only essential for motor control, but are also needed to accomplish perceptional tasks. Generally, the sensory input provided by an actual situation does not uniquely specify the situation. The information available might be incomplete, ambiguous or 
even contradictory. To find a unique interpretation of the sensory input, i.e., to solve the extra degree of freedom problem, the neuronal system has to engender an internal representation, a situation model which is constructed by application of both innate rules (e.g., Gestalt laws) and learnt knowledge.

What could such a body model look like? In the following, I briefly describe a neural network model which could be used for this purpose. To start with, the basic requirements to be fulfilled by such a "manipulable" body model need to be mentioned. To be a general purpose model it first of all must allow the representation of a system with redundant (extra) DoF. This is already the case when an arm with three joints is free to move in a two-dimensional plane. In this case the classical problem of inverse kinematics, namely to find three angle values such that the hand points to a given position in the two-dimensional plane, allows for many different solutions and the model must be able to select one of these solutions. To this end, the system should allow the representation of, in principle, all geometrically possible solutions, so that out of this infinite number of possibilities one can be selected. In order to adapt the model to the geometrical conditions of a given hardware system, it must also be able to cope with built-in constraints like, for example, joint limits. Furthermore, such a model must be capable of dealing with external constraints like, for example, holding the hand at a fixed angle if this is required by a specific task. Therefore, in order to allow for an explanation as simple as possible, we restrict the description to a model of only one part of the body, namely a three-joint arm moving in a two-dimensional plane (Fig. 1). It should, however, be stated that this model can be easily expanded to arbitrarily complicated geometries in 3D space (Kindermann \& Cruse, 2002).

A mechanical version of such a body model has been discussed by Mussa Ivaldi, Morasso, and Zaccaria (1988). These authors pointed out that the inverse kinematic problem could be easily solved by a mechanical model of the manipulator provided with springs to simulate the muscles, in the following way. By simply moving the tip of the (arbitrarily complicated) manipulator in the direction of the position of the intended target point, the joint angles of this mechanical model automatically find their appropriate values. The angle values of this model could then be used to control the real arm. Mussa Ivaldi et al. called this the "passive motion paradigm." In the form of the MMC net (Steinkühler \& Cruse, 1998), this idea has

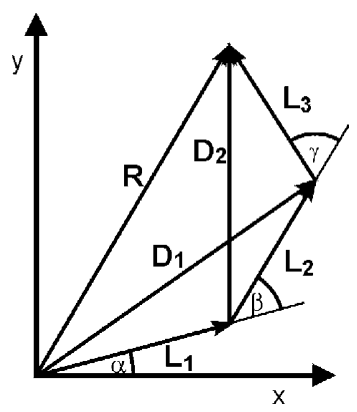

Fig. 1. An arm consisting of three segments described by vectors $\mathbf{L}_{1}, \mathbf{L}_{2}$, and $\mathbf{L}_{3}$, which are connected by the three joints. The joint angles are $\alpha, \beta$, and $\gamma$. The position of the endeffector is described by vector $\mathbf{R}$. Furthermore, two additional vectors $\mathbf{D}_{1}$ and $\mathbf{D}_{2}$, describing the diagonals, are shown. 


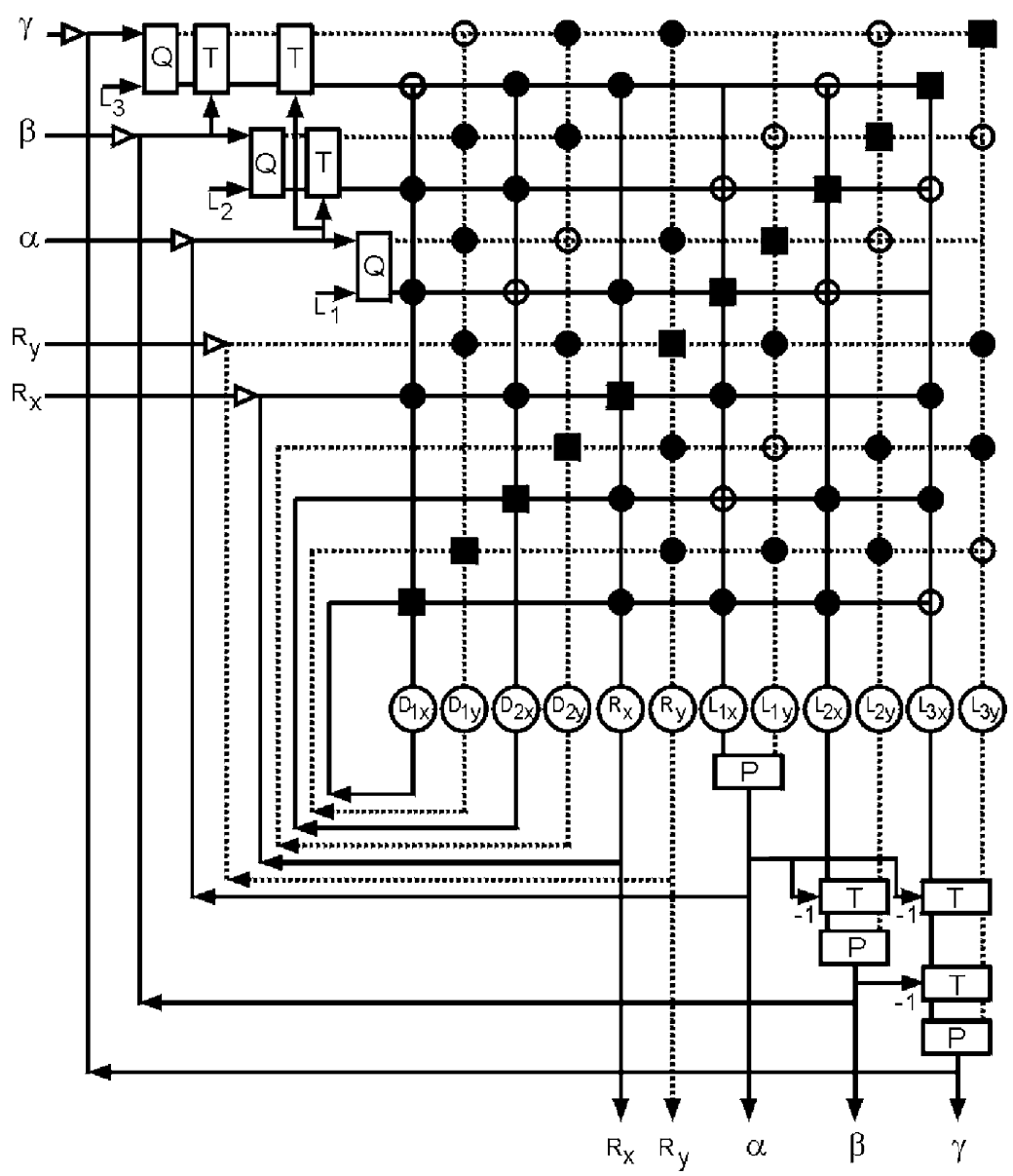

Fig. 2. Schematic drawing of the MMC net. The output values represent the components of the vectors $\mathbf{R}, \mathbf{L}_{1}, \mathbf{L}_{2}$ and $\mathbf{L}_{3}$ (lower line, see also Fig. 1). As input, for example, the new target position is given by the components $\mathbf{R}_{x}$ and $\mathbf{R}_{y}$ of the vector $\mathbf{R}$ (vertical column, left). Nonlinearities in the feedback loops (boxes T, P, and Q) are necessary to keep the segment lengths constant. The symbols (squares, open and filled circles) represent different connection weights (for details, see Steinkühler \& Cruse, 1998).

been implemented as an artificial neuronal network (Fig. 2). The architecture of this network is based on the principle often found in biology, that a given value is determined not only once, but simultaneously in several ways, thus exploiting redundancy. This principle is reflected by the name MMC which stands for mean of multiple computation. In general terms, this model consists of a recurrent network which relaxes to adopt a stable state corresponding to a geometrically correct solution, even when the input does not fully constrain the solution (Cruse \& Steinkühler, 1993). Any special constraints of the variables (e.g., joint limits) can be introduced. This recurrent network shows some similarities, but also important differences to Hopfield networks (for a detailed discussion, see Cruse et al., 1998). Hopfield nets show discrete attractor areas, whereas the MMC net has a smooth, bounded attractor space that represents all geometrically possible configurations of the arm. 

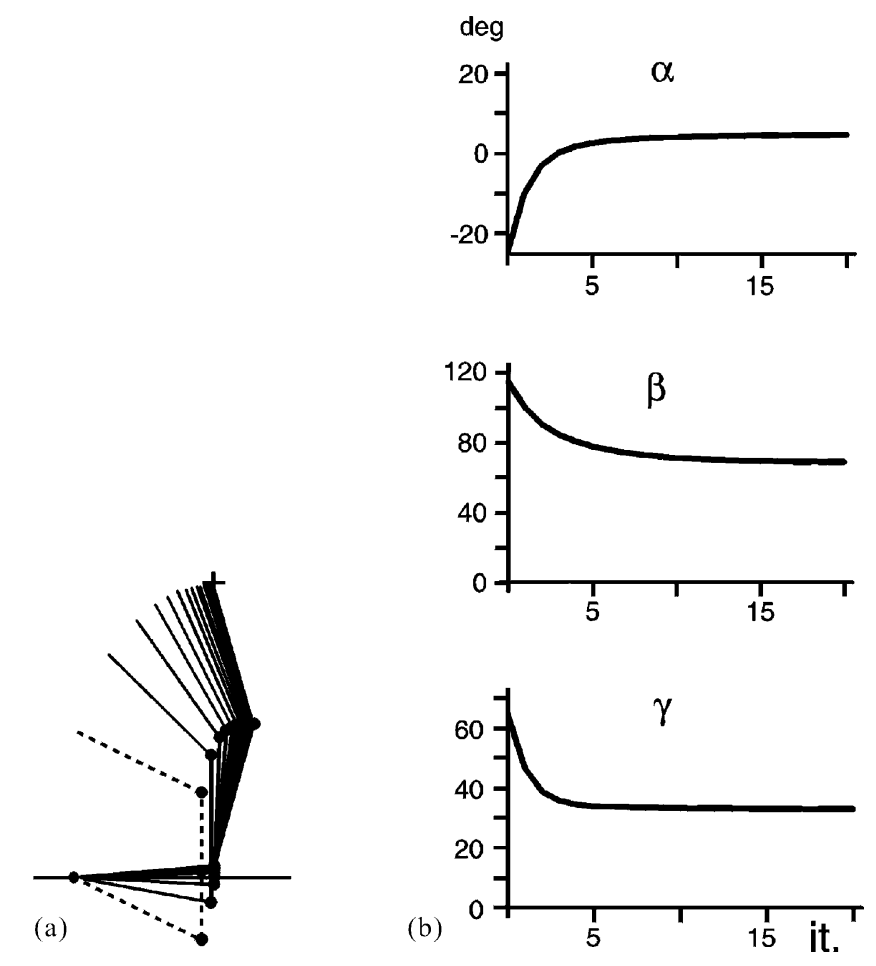

Fig. 3. Relaxation of a three-joint arm. (a) The arm moves from a starting configuration (- - -) to the goal position of the endeffector $(+)$. (b) The temporal changes of the three joint angles $\alpha, \beta$, and $\gamma$ during the relaxation. Abscissa is number of iteration cycles (it.).

Fig. 3 gives an example of how MMC solves an underconstrained inverse kinematic task. The movement of the manipulator starts from an arbitrary initial configuration (Fig. 3a, broken lines) with the task to point to a given target position (Fig. 3a, cross mark). The relaxation of the network is indicated by several intermediate arm configurations representing different iteration steps. As can be seen, the arm approaches the target point in an approximately exponential way. A special case is presented by the example in Fig. 4. Here, the coordinates of the endpoint cannot be reached by the manipulator because they specify a point outside its workspace. Nevertheless, the network tries to follow the desired coordinates as well as possible. The manipulator finally points in the direction of the desired endpoint coordinates. Although there is no solution to the problem, the network "does do its best."

In addition to some technical properties not mentioned here the MMC network shows the following general properties.

1. The net is able to control systems with redundant DoF. This includes the capability to choose one out of an infinite number of solutions possible in underconstrained situations. The system, for example, finds a geometrically possible solution even when only one value, for example, angle $\beta$ is prescribed. This solution can then be used to control the actual motor output. 
(a)

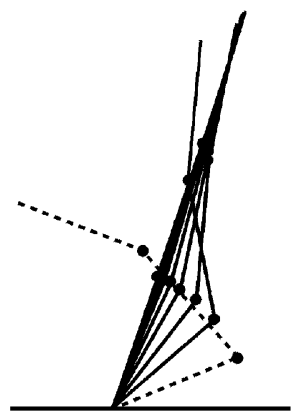

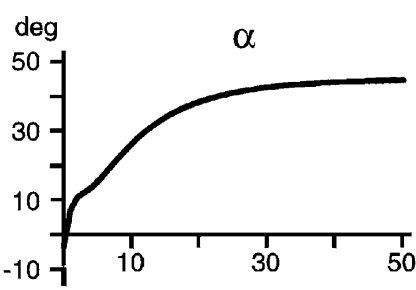

$+$

(b)

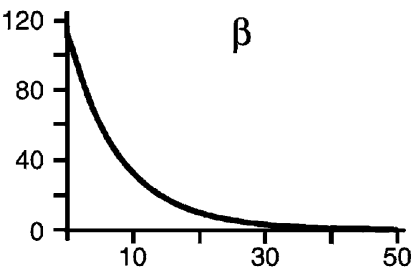

$\gamma$

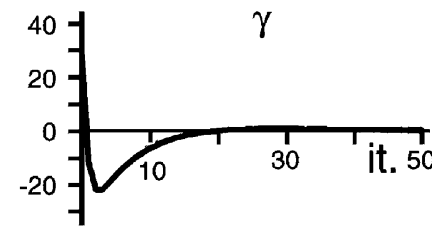

Fig. 4. Relaxation of the arm when the desired endpoint coordinates $(+)$ lie outside its workspace. Starting configuration of the arm is shown by broken lines (---). Only the arm configuration of every fifth cycle is shown in (a). (b) The temporal changes of the three joint angles $\alpha, \beta$, and $\gamma$ during the relaxation. Abscissa is number of iteration cycles (it.).

2. The dynamic properties of the net show qualitative parallels to Fitts's law (1954), which describes a tradeoff between speed and exactness of a targeting movement. As shown in Fig. 3, the manipulator meets the desired end point the better, the longer the net has time for relaxation. If, however, time is short, an early, but then necessarily only approximate solution could be read out from the network to control the motor output. Therefore, if the network is interpreted as to provide the endpoint of the movement, but velocity of the movement to be given by a separate input to the motor system, a behavior according to Fitt's law can be observed. The velocity of the movement might, however, also be influenced by the network itself through variation of the damping parameters (Fig. 2, weights along the diagonal depicted as squares). For higher damping values, movement speed is slow and the errors are the smaller the longer the net has time for relaxation, whereas for small values of the damping parameters, the simulated movement is fast and shows considerable oscillations around the target position, leading to larger errors. Both effects contribute to a behavior corresponding to Fitt's law.

3. The network could be considered as forming a "dynamic map" of the arm, because it is able to represent all geometrically possible configurations. However, it requires a very small number of units compared to the usual topological, e.g., Kohonen type mappings (e.g., Walter \& Ritter, 1996). 
4. Another aspect interesting for the interpretation of possible functions of neurons known from electrophysiological recordings is that, in an MMC type system, the internal connections cannot be labeled as either motor or sensory elements. As summarized by Fuster (1995, pp. 134-137) there was a discussion whether cells found in area 7 of a monkey which are excited when the monkey was looking at or was reaching to a given target should be termed motor or sensor neurons. Application of the MMC type network overcomes the sensorimotor ambiguity, because in this view a classification of neurons as more sensory or more motor is not sensible. In a similar way, this holds for the "bimodal neurons" described by Iriki, Tanaka, and Iwamura (1996) in the caudal postcentral gyrus which code body-centered extrapersonal visual space (see also Sakata et al., 1997). Furthermore, the below mentioned "mirror neurons" could be explained this way. Another example of units that cannot uniquely be attributed to either the motor system or the perceptual system are the "decision neurons" (Kast, 2001).

5. The system solves the problem of sensor fusion, occurring when different sensory inputs represent the same value. As an example, in the case of the three-joint arm, the endeffector coordinates ( $\mathbf{R}_{x}$ and $\mathbf{R}_{y}$ in Figs. 1 and 2 ) could be given by visual input, the angles $\alpha, \beta$, and $\gamma$ by mechanoreceptor inputs. These different values are integrated within this holistic system. In this way, the MMC net concatenates both the visual and the mechanoreceptively given spaces to form a common, dynamic representation.

6. Similarly, an MMC type network may provide a quantitative basis for the phenomena called "Gestaltkreis" by Weizsäcker (1950), "action-perception cycle” by Arbib (1981), or representation-execution continuum (Jeannerod, 1994, 1997). In a corresponding way, Prinz (1997), following Lotze's (1852) early ideas on the ideomotor principle, proposes a "common coding of perception and action." He states that to perform an action, a "stimulus code" has to be related to an "action code" the latter consisting of a "goal code" and a "motor code." This triplet, forming an entire system, has been termed "intention code" by Prinz (1997). Experimental evidence supporting the common coding hypothesis is further summarized by Decety and Grèzes (1999), in particular, for the case when the subject intends to imitate observed movements. Thus, the MMC net can be regarded as a possible realization of the common coding of stimulus and goal code postulated by these ideas.

\section{Planning}

This neuronal model can, as described, be used as a network to control movement, even in ill-posed situations. Apart from this capability of directly controlling complex motor systems, i.e., motor systems with many serial DoFs, the network can be interpreted in another way: When being disconnected from the motor output and parts of the sensory input, it can be used for the internal representation of the movement of an arm without actually performing the movement. In this way, the network can be considered a basis of imagining a movement. Jeannerod (1999) summarizes a number of different results of his group and of other authors supporting the hypothesis that motor imagery and motor performance are based on similar neural substrate. This is further supported by recent results of Lotze et al. (1999) who found that during both 
actual and imaginal movement the premotor area and supplementary motor area (SMA) are active in a similar way. Furthermore, it has been reported (Rizzolatti \& Arbib, 1998) that in the case of mere observation of an action, a strong inhibitory influence has been found which selectively inhibits those motoneurons in the spinal chord which would be involved in the execution of the observed action. Similarly, specific inhibitory influences during imagined movement has been observed (Jeannerod, 1999). Therefore, the corresponding motor output appears to be actively inhibited during imagination of a motor act or during observation of an action.

Thus, when being disconnected from the motor output, the MMC network might, as mentioned, be used as a basis to imagine movements and therefore be regarded as a possible basis for a "mental model" of the body. Motor imagery is similar to mental rotation (Shepard \& Metzler, 1971; Georgopoulos, Lurito, Petrides, Schwartz, \& Massey, 1989), but more difficult to the extent that, in the case of controlling a redundant manipulator, the geometrical relationships between the body parts are not fixed, but, in general, have to be changed relative to each other to find a solution. A mental model might also be used for the internal search for the solution of a motor task. Therefore, the MMC network represents a circuit which could serve as a neuronal basis for motor performance, motor imaging and motor planning. Using an expression of Gallese (2000) this network comprises the ability of a motor system to "master its own representation.” Following O'Regan and Noë (2002), this network allows “the mastery of the relevant sensorimotor contingencies." It is important to say that the network does not represent the possible arm configurations in the form of a look-up table, but represents the underlying mechanism instead. Following Byrne's (2000) terminology, the model permits a "representational understanding of mechanism." The network being able to construct a situation model based on sensory information, yet possibly incomplete, could well provide a basis for the "remembered present" — a most imaginative term coined by Edelman (1989).

Thus, the MMC model can be used for the manipulation of knowledge-in this case geometrical knowledge concerning the own body - it can "play around" with different possible solutions until it has found a (new) solution for a given task. This means that, according to the definition used here (see also McFarland \& Bösser, 1993), this network may be regarded as a basis for cognition. Should this search for a solution be described as a "decision" performed by the network? There are two kinds of situations leading to a decision: one is described by a continuous decision space as it occurs with the control of the arm's movement, the other refers to a discrete decision space such as the decision between fight and flight. A discrete decision situation, however, can also occur in the arm example when the movement of the hand is obstructed by an obstacle and a decision has to be made whether to pass the obstacle on its left or its right. Another example is given by a reaching task in which an object has to be pointed at with either the right or the left hand (Kim \& Cruse, 2001). As far as discrete decisions are concerned, it is usually assumed that the different possible solutions have to be tested and stored separately, so that, when comparing the different solutions, a decision can finally be made. This method corresponds to the deliberation procedure as described by Sloman (2000) who proposes the distinction between reactive and deliberative systems, where I distinguish between reactive and cognitive systems. Such a deliberation procedure, using a more or less pruned search tree (Aleksander, 2000), can only be applied to the case of a continuous decision space with difficulty, because it is almost improbable that all 
possible solutions could be stored that finally lead to a decision. In this case, the application of a system with a continuous attractor space appears to be more appropriate. However, also discrete decision situations can be solved by a dynamic system without explicit storage of the discrete solutions (e.g., Hopfield \& Tank, 1985, or, for the above mentioned decision between right and left hand, Kim \& Cruse, 2001). In such systems as in the MMC-net, which may be considered as networks underlying Aleksander's "depiction," manipulation of knowledge occurs without processing of symbols and explicit search tree. Therefore, the term cognition is used here as the more general term (see also Tani, 1998) for this type of "mental activity." As will be argued later, specific continuous dynamic systems that represent the underlying mechanism in a holistic way appear necessary to form the basis for subjective experiences which, I think, is not possible for deliberative procedures based on search trees. Another way to deal with this question is the following. Referring to a decision as a discrete one appears only to be appropriate as an a posteriori description, i.e., when looking at it after the fact. The actual neuronal activity has to be performed in a continuous situation (see also Edelman \& Tononi, 2000, who consider the necessity to select a solution out of a very large number of possibilities as an important condition for consciousness to emerge).

An MMC type network can therefore be considered as a possible basis for a sensorimotor hypothesis of cognition. A common basis for low-level motor, and higher level cognitive processes has also been postulated by Smith and Shadmehr (2000) who address the basal ganglia as to play an important role in this context, however, also posterior parietal cortex, cerebellum and motor cortices are discussed to contribute to an internal body model (Desmurget \& Grafton, 2000; Decety \& Grèzes, 1999). An extremely elaborate proposal has been made by Damasio (1999). All the brain areas he is listing as possible basis for what he names core consciousness (i.e., sensorimotor cortex, cingulate cortex, thalamus and superior colliculi) may be regarded as a possible anatomical basis where these networks may be found.

Several researchers argue along similar lines. Fuster summarizes his view stating that thinking is some kind of imagined movement (Fuster, 1995). Calvin (1996) argues that thinking is movement which has not yet taken place (see also Jeannerod, 1999). Ito (1993) states that "in thought, ideas and conceptions are manipulated just as limbs are in movement." Jeannerod (1999) expands this view of a holistic action-perception system by stating that an observer understands an observed action "whenever he/she becomes able to simulate it and to share the experience of the person who performs the action." And he summarizes an impressive amount of own work and of others stating that "such processes as intending, imaging, observing/imitating and performing an action share, at least in part, common structural and functional mechanisms." In other words, even perception might be based on the same system. Therefore, I propose that an MMC-like structure may represent the basis for a perceptual system, too (for a stimulating discussion of a tight connection between perception and the motor system see also Gross, Heinze, Seiler, \& Stephan, 1999). This agrees with the observation of the already mentioned "mirror neurons." As reviewed by Rizzolatti and Arbib (1998), specific neurons in premotor areas are activated if an individual is about to perform an action or observes another individual performing this action. These neurons have therefore been called "mirror neurons." These neurons appear to be tightly coupled to the motor system and are interpreted to provide the basis for a system that allows for action understanding (Gallese, 2000). In that sense such an internal model might help to live in a social environment where the activities of conspecifics 
have to be recognized and understood. Recent support for a tight connection between what is traditionally divided in a perception system and a motor system is given by Buccino et al. (2001). Human subjects are allowed to look at videos that show kicking movements of a leg, grasping movements of an arm, or chewing movements of a mouth. Imaging studies revealed strong excitation of the corresponding motor areas. When the observed movement was coupled with an adequate object, a ball, a cup, or an apple, additional excitation has been found in the parietal cortex. Interestingly, patients with specific prefrontal lesions compulsively imitate gestures or even complex actions performed by the experimenter (Lhermitte, Pillon, \& Serdaru, 1986, cited from Gallese \& Goldman, 1998). This can be interpreted as an impairment of the inhibition of the connection between the internal model and the motor output.

Following these ideas, an internal body model like the MMC net could be regarded as representing the basis for a simple form of thinking. This may be made more plausible when we consider the possibility that the body model might be expanded by including external objects like tools, for example, a stick held by the hand. A plausible biological basis for this may be given by results of Iriki et al. (1996) who found bimodal neurons in the caudal postcentral gyrus with receptive fields representing the position of the hand in space. During tool use, their visual fields were altered to include the entire stick held by the hand of the monkey. In the MMC net, such an expansion is easily possible by recruiting additional neurons representing the tool. In this way, the MMC net might serve not only as an internal body model in a strict sense, but could easily be extended to form a simple, egocentric world model. I would like to underline here that a body model already constitutes a world model insofar as the body is part of the world. Moreover, the MMC network does not only represent the geometry of the body. The net also contains a small part of the external world in the form of the vector $\mathbf{R}$ (Figs. 1 and 2) which represents the position of an external object. Furthermore, the MMC net can easily be expanded to represent additional geometrical positions in space. For example, it can be used to represent different landmarks in order to control landmark navigation (Cruse, submitted) or, to represent the position of a group of conspecifics. To allow for an allocentric perspective, separate structures may be necessary (Jeannerod, 1999; Vogeley et al., 2001).

\section{Internal perspective}

Some people assume that "true cognition" is only possible for systems with an internal perspective or a first person's view (also termed phenomenal consciousness, see e.g., Chalmers, 1996). Simple reactive systems are assumed to have no internal perspective. Humans, on the other hand, are systems that are able to have an internal perspective. This distinction is not immediately obvious to everybody, probably because having an internal perspective is so much identical with being oneself that it is difficult to imagine that having an internal perspective is a separable property. To make this clearer, I would like to introduce two terms already used earlier (Cruse, 1999), namely the terms of HIP system and NIP system. Apparently, in this world we can distinguish between two types of systems. There are systems, that definitely include humans, and most probably many other animals, that are capable of subjective experience, sometimes also called awareness or internal perspective. For short they shall be called HIP systems (Having an Internal Perspective). There are other systems, such as a pencil, a stone, 
or a clockwork, that most probably do not have this ability. I shall call them NIP systems (Not having an Internal Perspective). They do not feel anything. This is at least, what people usually take for granted. Although we cannot be totally sure about it, I shall adopt this view to simplify the following discussion. In many cases, for example, in an insect, we cannot decide whether it belongs to the NIP systems that act like a reflex machine, or a clockwork, or whether it belongs to the second type, the HIP systems, and consequently has subjective experiences. Such a decision is the more difficult, the more the brain under view differs from a human brain. (For an excellent description of this topic, see Damasio (1999). In his terminology, a system has to be endowed with at least "core consciousness" to belong to the realm of HIP systems. Probably Edelman and Tononi's (2000) "primary consciousness" has a similar meaning).

The following example may serve to make the distinction between HIP and NIP systems more obvious. Imagine we were able to look inside the brain of a subject and measure all the interesting neuronal activities, for example, when the subject judges the color of a, say green, pencil. In this way, all details of the subject's neuronal activities when seeing "green" could be determined. On the other hand, the subject seeing green does not see these neuronal activities, but experiences seeing green. The latter is called the subjective quality of experience. The content of this subjective, or first-person, perspective is only accessible to the person himself or herself. Nobody other than myself can judge how I see green. The characteristic of subjective experience becomes even more obvious in the case of pain. We might consider all neuronal activities that occur when a subject's skin is stimulated by a needle. One might, in principle, even examine one's own action potentials, if oneself is the subject of this experiment. But the experience when considering all these neuronal activities is completely different from the pain one experiences at this moment. Thus, self-observation tells us that there are systems, namely humans, that can experience an internal perspective. On the other hand, intuition tells us that there are other systems, like a stone or a simple machine (including some clever present-day robots) that may not have such an internal perspective.

Interestingly, there are at least three cases that mark a transition from a system without internal perspective, a NIP system, to a HIP system, i.e., a system capable of an internal perspective. At least once during biological evolution, somewhere between the state of the simple protozoans and the state of the human being, this change must have come about. Such a transition also occurs during the ontogeny of each individual human. A, say, four-cell human embryo most probably does not yet have the ability to experience, because we assume that a specific neuronal system is necessary to have subjective experience. So, somewhere during ontogeny this change must occur. Furthermore, our everyday change from (dreamless) sleep to the awake state shows such a transition. This means that even the brain of an adult human can, to some extent intentionally, switch between these two states.

Using this distinction, one might ask whether there is still a principal difference between HIP systems and systems having the property to be cognitive in the sense used here, i.e., to be able to plan ahead? In other words, do HIP systems require additional circuits beyond those discussed above? To approach this question we should first consider what could be said concerning the content of our subjective experience.

According to Metzinger (2000), subjective experience does not reflect our direct sensory input, but relies on the content of a construct. This view is supported by a number of phenomena which here might only be mentioned briefly: we do not experience the actual image projected 
on our retina, but have the impression of a stable space. We see the 2D Necker cube as a 3D figure. When we try to explore the world by a hand held stick as mentioned above we soon have the impression of feeling the tip of this stick although there are definitely no sense organs. A mechanical stimulation at the skin is felt at the stimulated site of the body, although the neural processes forming the basis of this experience occur in the brain. The view that what we subjectively experience is a construct is even more obvious when considering phantom sensation where no sense organs exist. Other examples supporting this view are hemineglect, confabulation, alien hand syndrome or mania. O'Regan and Noë (2002) summarize impressive cases of "change blindness." Libet, Wright, Feinstein, and Pearl (1979) experiments show that an about $500 \mathrm{~ms}$ excitation of the cortex is necessary to experience a subjective sensation, but there is a back projection in time such that we have the impression that the experience occurs together with the beginning of the stimulation. Metzinger summarizes these results by saying that we confuse the content of our subjective world model with the real world.

These mental constructs form a subjective world model. Above I have already discussed recurrent networks that are assumed to represent a world model. Therefore, I would like to speculate that the mental constructs forming a subjective world model are based on the recurrent networks discussed earlier. Is it possible to confirm this statement? Probably the only possible way to approach this question is an indirect one. We have to try to find parallels between observations concerning subjective experiences of systems such as human beings, i.e., so-called HIP systems, with properties of the neuronal system. The more parallels we find, the more the hypothesis is supported. For example, the MMC net shows parallels to Libets experiments, if we assume further that an approximate relaxation of the recurrent network is a necessary condition to reach the state of subjective experience, that is the HIP state: relaxation to a stable attractor takes some time, and Libet's experiments reveal that some time is necessary for a stimulation to elicit subjective experience.

According to Damasio (1999) core consciousness comes in a pulse-like fashion, and consists of three states unfolding in time: the initial state corresponds to the actual state of the organism. The second stands for the arrival of an object and the third for a reaction that results in a modified state of the organism (Damasio, 1999, p. 168). The latter state is engendering the phenomenon of subjective experience. The MMC net proceeds in the same way. At the beginning, the body position it represents has to be updated using sensory input to correspond to the actual body position. Second, an input is provided representing an external object that, in Figs. 1 and 2, corresponds to vector $\mathbf{R}$, and third, after some time necessary for relaxation, the internal model has changed its position which may, or may not, be used to control the movement of the body. According to my above speculation, successful relaxation corresponds to this positional change becoming subjectively experienced. Correspondigly, Damasio states that "we become conscious, then, when. . an organism has been changed by an object...." However, unlike Damasio's assumption there is no two-stage representation, no "re-representation" necessary, when using such a recurrent network. In the MMC network there is no separate representation of object and of body. Rather, both are represented in a holistic manner within one network. O'Regan and Noë give a nice example in order to explain the occurrence of subjective experience by asking what is it like to drive a Porsche: "The particular experience of Porsche-driving comes from the typically Porsche-like give-and-take between you and the car when you drive the Porsche" (O’Regan \& Noë, 2002). This means there is a continuous comparison between 
the prediction of the internal "Porsche-driving" model and the sensory input. If both fit, the recurrent network model will relax and the lucky subjective experience of Porsche-driving will occur. Edelman and coworkers (e.g., Edelman \& Tononi, 2000) have investigated a series of model studies based on perceptual phenomena and have used a different type of network. However, they come to very similar general conclusions: to reach consciousness, a recurrent network is necessary that can select one solution out of a very large number of possibilities. This solution is influenced by the sensory input and requires some time possibly corresponding to the psychological refractory period. Edelman and Tononi (2000) call these networks "functional clusters" and offer most fascinating insights, when discussing how such clusters might be imbedded in a larger framework, a topic not addressed here further (for an early model referring to this topic, see Cruse, 1979).

Some further examples show parallels to the properties of the MMC net, as well. Neumann and coworkers (Ansorge, Klotz, \& Neumann, 1998) performed a series of experiments where subjects had to press a button when a specific visual stimulus was perceived. In some experiments the stimulus had been masked so that the subjects report not having seen the stimulus, but they nevertheless react to the presentation of the stimulus as if it had been perceived. This means that subjective experience is not necessary for the control of an action. The authors discuss several models to explain this result. Their favorite model, the "late dissociation view" assumes a process with dynamic properties: this hypothetical process can drive the motor output already in an early phase, but requires some time of further processing until the content of the process reaches a conscious level. Masking is assumed to interrupt this activity. An MMC type network might well be the basis of this phenomenon, because, as mentioned above, the motor output may be active already in an earlier state of relaxation, whereas final relaxation, i.e., the mechanism hypothetically leading to subjective experience, can be interrupted by a new input given to the system. This view of a tight connection between the motor control system and the system responsible for subjective experience fits to the observation of Ekman (1992) who states that the activation of motor commands for facial expressions showing happiness or sadness, for example, are necessarily followed by the corresponding subjective experiences.

A spectacular case has been reported by Ramachandran, Dogers-Ramachandran, and Cobb (1995). In a patient suffering from phantom sensation in the amputated left arm, a lively subjective experience of moving this nonexisting left arm could be elicited when the intact right arm was both actively moved and seen in a mirror such that the mirror image of the right arm appeared at the position where the left arm would have appeared if it had been intact. This observation could be interpreted in the following way: usually the model of the arm is in a relaxed and therefore subjectively experienced state, for example, pointing straight to the left. No change of the state is possible because there is no sensory input. In the experiment, the visual input providing information concerning the position of the arm is sufficient to activate the model of the left arm and that this activation in turn leads to a new subjective experience. Furthermore, it is interesting that the phantom sensation can disappear when the patient uses the mirror for several weeks. This indicates that at least part of the body model can be unlearned (Ramachandran \& Blakeslee, 1998).

The Pinnochio illusion can be experienced when the biceps muscle of an subject's arm is vibrated while the hand is grasping the nose (Lackner, 1988). In this situation the nose appears to be elongated up to $30 \mathrm{~cm}$. Vibration stimulates the muscle spindles such that the elbow 
joint appears to be more extended. As the nose is still grasped by the hand, this incongruent situation leads to a subjective experience which can be interpreted as to represent some kind of compromise between the different, non-matching sensory signals. A corresponding behavior can be found in the MMC model as shown in Fig. 4 (see also Cruse et al., 1998, Fig. 3, which shows the elongation of body segments). In this incongruent situation the network, too, relaxes to some kind of compromise state. A discussion to what extent this model may further agree with the phenomena described by philosophers as semantic transparency and perspectivalness can be found in (Cruse, 1999).

Experiments of Povinelli and collaborators (e.g., Povinelli, Landry, Theall, Clark, \& Castille, 1999) provide information concerning the development of body models in human children. These suggest that internal world models can be activated by sensory input-as revealed in Ramachandran's patient - already in 2-year-old children and in chimpanzees. When these children or chimpanzees are filmed by a video camera and simultaneously look at the monitor, they apparently recognize themselves. However, this is no longer the case when there is a time delay of about $3 \mathrm{~min}$ between the actual behavior and the video. In this latter situation self-recognition is only possible for children of age 4 or 5 , but not for the apes. This suggests that the ability to use the body model independently of the actual sensory input is developed in children between age 2 and 5 .

These observations do of course in no way prove that an MMC type network is a necessary condition to form a HIP system. They allow, however, the suggestion that the neuronal system that forms the basis of the internal model does not consist of a perceptron-like feedforward system. Such a look-up table cannot represent the temporal dynamics nor can it represent the underlying mechanisms of the real world system, in our example the three segment arm. Although such an arm could be represented by a multi-layer perceptron (Brüwer \& Cruse, 1990), a perceptron could not easily be adapted to any arbitrary external constraint, but required a special training session for each new type of constraint. This is not necessary for a system that does not represent the input-output relations in the form of a look-up table, but the underlying mechanism. Therefore, two hypotheses are formulated here. One is that our internal world model is based on a recurrent network with attractor dynamics that is able to represent the underlying mechanisms. The MMC network only shows one possible realization of such a system. The second hypothesis claims that part of the content of this world model can be subjectively experienced if the net is relaxed to a sufficient degree. Flohr (2000) argues that if such a system develops "higher-order self-reflexive representations," this is not only a necessary, but also a sufficient condition to be a HIP system, i.e., for the occurrence of subjective experience. If this was true, we had to conclude that artificial systems could exist that have subjective experience, too. According to this idea, a system, like "Deep Blue," however, able to defeat a world chess champion, cannot reach the state of a HIP system, because it contains a planning system that does not represent the mechanisms underlying chess in a holistic way.

Finally, it should be mentioned that this model might be considered to explain how the hardware basis of our ability to have an internal perspective might look like, but it does in no way explain the really "hard problem" (Chalmers, 1996) namely to say what enables a physical system to have subjective experience. In other words, these considerations do not explain why some neuronal activities are accompanied with subjective experience whereas 
others are not. This question has not been solved yet. It has, however, been argued (Cruse, 1999) that an astonishingly simple solution will be reached in a way similar to how the question of what life is has been answered. Today we know the essential mechanisms that make up a living system. Therefore, nobody in science cares for a vis vitalis required by the vitalists at the beginning of the 20th century as essential for life to exist. Similarly, in the future most people will be satisfied with the idea that just some specific mechanisms make up a HIP system.

\section{Conclusion}

Behavior may be controlled by reactive systems. In a reactive system the motor output is exclusively driven by actual sensory input. Such a system could possibly be expanded by being equipped with internal states. In this case the actual sensory input can be treated differently depending on the system's actual state. Generally, these internal states are also sensorily driven, but this happens on a longer time scale. A further expansion of such reactive systems might include the introduction of learning mechanisms. Even with these expansions the system might still be termed a reactive system in a broad sense because its behavior is, on a short or a long term basis, controlled by sensory input. There are a number of concrete examples in the form of robots which show that such reactive architectures allow the construction of systems with a considerable degree of autonomy.

However, in order to control bodies with a high number of DoFs, an internal representation of the body is assumed to be necessary. An artificial neural network is described which might form the basis of such an internal representation. Minor changes suffice that such a system can also be used as a basis for a planning system and therefore can become a cognitive system. This agrees with the proposal of several researchers that thinking can be understood as imagined movement (see also S. Freud's "Probehandeln"). Anecdotical evidence supporting this view is given by biological examples for the control of complex kinematics: beside primate grasping the control of the trunk by elephants, manipulation of objects with legs and the beak by parrots or, to take an invertebrate example, the movement of the arms of an Octopus. All these animals appear to have considerable cognitive abilities. Dolphins appear not to be an example supporting this sensorimotor hypothesis of cognition, because they do not have a motor system with a particularly high number of DoFs (compared to other vertebrates). However, they live in social groups and might therefore have found a completely different way to develop a world model which is able to represent the spatial position of the members of the group. As indicated, an MMC type model may also be the basis for such a world model.

Concerning the evolution of cognitive systems, the view proposed here means that a cognitive system might have been evolved on the basis of an already existing neuronal system necessary for the control of a geometrically complex body, i.e., a body with serially redundant DoFs. Cognition is possible when the connections between the neuronal system and the motor system can be disconnected. Therefore, according to this proposal evolution of cognition is an example of "exaptation" (Byrne, 2000). This means that the evolutionary development of cognitive abilities may not have required the invention of completely new neuronal systems, but rather the ability to disconnect already existing structures. Thus, reactive and cognitive systems should 
not be considered as logically exclusive ones. Cognitive systems should rather be regarded as a special form of reactive systems like humans are a special form of animals. Concerning future research strategies, these considerations suggest that the understanding of how to control a complex kinematic system may be a prerequisite to understanding the functioning of cognitive systems.

\section{Acknowledgments}

I would like to thank Beate Cruse for many helpful discussions, Manuela Lenzen for hints to literature as well as Stan Franklin, Peter Mandik and another anonymous referee for very valuable comments. This work was supported by DFG grant $\mathrm{Cr}$ 58/9-3.

\section{References}

Aleksander, I. (2000). Brain inspired computing: The next 50 years of Artificial Intelligence. RSA Journal, 4, 74-78. Ansorge, U., Klotz, W., \& Neumann, O. (1998). Manual and verbal responses to completely masked (unreportable) stimuli: Exploring some conditions for the metacontrast dissociation. Perception, 27, 1177-1189.

Arbib, M. A. (1981). Perceptual structures and distributed motor control. In V. B. Brooks (Ed.), Handbook of physiology: Nervous systems (Vol. 2, pp. 1448-1480). Bethesda, MD: American Physiology Society.

Atkinson, A. P., Thomas, M. S. C., \& Cleeremans, A. (2000). Consciousness: Mapping the theoretical landscape. Trends in Cognitive Sciences, 4, 372-382.

Beer, R. D., Chiel, H. J., Quinn, R. D., \& Ritzmann, R. E. (1998). Biorobotic approaches to the study of motor systems. Current Opinion in Neurobiology, 8, 777-782.

Bernstein, N. A. (1967). The co-ordination and regulation of movements. Oxford: Pergamon Press.

Brooks, R. A. (1991a). Intelligence without reason. Proceedings of the 12th International Joint Conference on Artificial Intelligence, Sydney, Australia, August 1991, pp. 569-595.

Brooks, R. A. (1991b). New approaches to robotics. Science, 253, 1227-1232.

Brooks, R. A. (1997). From earwigs to humans. Robotics and Autonomous Systems, 20, 291-304.

Buccino, G., Binkofski, F., Fink, G. R., Fadiga, L., Fogassi, L., Gallese, V., Seitz, R. J., Zilles, K., Rizolatti, G., $\&$ Freund, H.-J. (2001). Action observation activates premotor and parietal areas in a somatotopic manner: An fMRI study. European Journal of Neuroscience, 13, 400-404.

Brüwer, M., \& Cruse, H. (1990). A network model for the control of the movement of a redundant manipulator. Biological Cybernetics, 62, 549-555.

Byrne, R. W. (2000). Evolution of primate cognition. Cognitive Science, 24, 543-570.

Calvin, W. H. (1996). How brains think. New York: Basic Books.

Chalmers, D. J. (1996). The conscious mind. New York: Oxford University Press.

Cruse, H. (1979). Modellvorstellungen zu Bewußtseinsvorgängen. Naturw Rdschau, 32, 45-54.

Cruse, H. (1999). Feeling our body-The basis of cognition? Evolution and Cognition, 5, 162-173.

Cruse, H. (submitted). The explanatory power and limits of simulation models in the neurosciences. In P. Machamer, R. Grush, \& P. McLaughlin (Eds.), Theory and method in the neurosciences (pp. 138-154). Pittsburgh: University of Pittsburgh Press.

Cruse, H., Brüwer, M., \& Dean, J. (1993). The control of three- and four-joint arm movement: Strategies for a manipulator with redundant degrees of freedom. Journal of Motor Behavior, 25, 131-139.

Cruse, H., Kindermann, T., Schumm, M., Dean, J., \& Schmitz, J. (1998). Walknet—A biologically inspired network to control six-legged walking. Neural Networks, 11, 1435-1447.

Cruse, H., \& Steinkühler, U. (1993). Solution of the direct and inverse kinematic problem by a unique algorithm using the mean of multiple computation method. Biological Cybernetics, 69, 345-351. 
Cruse, H., Steinkühler, U., \& Burkamp, Ch. (1998). MMC—A recurrent neural network which can be used as manipulable body model. In R. Pfeifer, B. Blumberg, J.-A. Meyer, \& S. W. Wilson (Eds.), From animals to animats (Vol. 5, pp. 381-389), Cambridge, MA: MIT Press.

Damasio, A. R. (1999). The feeling of what happens. Body and emotion in the making of consciousness. New York, San Diego, London: Harcourt Brace \& Company.

Dean, J., Ritter, H., \& Cruse, H. (Eds.). (2000). Prerational intelligence: Interdisciplinary perspectives on the behavior of natural and artificial systems. Dordrecht: Kluwer Academic Publishers.

Decety, J., \& Grèzes, J. (1999). Neural mechanisms subserving the perception of human actions. Trends in Cognitive Sciences, 3, 172-178.

Desmurget, M., \& Grafton, S. (2000). Forward modeling allows feedback control for fast reaching movements. Trends in Cognitive Sciences, 4, 423-431.

Dörner, D. (1999). Bauplan einer Seele. Reinbek: Rowohlt.

Edelman, G. M. (1989). The remembered present: A biological theory of consciousness. New York: Basic Books.

Edelman, G. M., \& Tononi, G. (2000). A universe of consciousness. How matter becomes imagination. New York: Basic Books.

Ekman, P. (1992). Facial expression of emotion: New findings, new questions. Psychological Science, 3, 34-38.

Fitts, P. M. (1954). The information capacity of the human motor system in controlling the amplitude of movement. Journal of Experimental Psychology, 47, 381-391.

Flohr, H. (2000). NMDA receptor-mediated computational processes and phenomenal consciousness. In T. Metzinger (Ed.), Neural correlates of consciousness (pp. 245-258). Cambridge, MA: MIT Press.

Fuster, J. M. (1995). Memory in the cerebral cortex. Cambridge, MA: MIT Press.

Gallese, V. (2000). The acting subject: Toward the neural basis of social cognition. In T. Metzinger (Ed.), Neural correlates of consciousness (pp. 324-333). Cambridge, MA: MIT Press.

Gallese, V., \& Goldman, A. (1998). Mirror neurons and the simulation theory of mind-reading. Trends in Cognitive Sciences, 2, 493-501.

Georgopoulos, A. P., Lurito, J. T., Petrides, M., Schwartz, A. B., \& Massey, J. T. (1989). Mental rotation of the neuronal population vector. Science, 243, 234-236.

Gross, H. M., Heinze, A., Seiler, T., \& Stephan, V. (1999). Generative character of perception: A neural architecture for sensorimotor anticipation. Neural Networks, 12, 1101-1129.

Hopfield, J. J., \& Tank, D. W. (1985). "Neural" computation of decisions in optimization problems. Biological Cybernetics, 52, 141-152.

Iriki, M., Tanaka, Y., \& Iwamura, Y. (1996). Coding of modified body schema during tool use by macaque postcentral neurons. Neuroreport, 7, 2325-2330.

Ito, M. (1993). Movement and thought: Identical control mechanisms by the cerebellum. Trends in Neurosciences, $16,448-450$.

Jeannerod, M. (1994). The representing brain: Neural correlates of motor intention and imagery. Behavioral Brain Sciences, 17, 187-245.

Jeannerod, M. (1997). The cognitive neuroscience of action. Oxford: Blackwell.

Jeannerod, M. (1999). To act or not to act: Perspectives on the representation of actions. Quarterly Journal of Experimental Psychology, 52A, 1-29.

Kast, B. (2001). Decisions, decisions.... Nature, 411, 126-128.

Kim, D.-H., \& Cruse, H. (2001). Two kinds of body representations are used to control hand movements following tactile stimulation. Experimental Brain Research, 139, 76-91.

Kindermann, T., \& Cruse, H. (2002). MMC-A numerical approach to the kinematics of complex manipulators. Mechanism and Machine Theory, 37, 375-394.

Lackner, J. R. (1988). Some proprioceptive influences on the perceptual representation of body shape and orientation. Brain, 111, 281-297.

Lanz, P., \& McFarland, D. (2000). Philosophical perspectives on representation, goals, and cognition. In J. Dean, H. Ritter, \& H. Cruse (Eds.), Prerational intelligence: Interdisciplinary perspectives on the behavior of natural and artificial systems (pp. 247-263). Dordrecht: Kluwer Academic Publishers.

Lhermitte, F., Pillon, B., \& Serdaru, M. (1986). Human autonomy and the frontal lobes: I. Imitation and utilization behavior: A neuropsychological study of 75 patients. Annals of Neurology, 19, 326-334. 
Libet, B., Wright, E. W., Feinstein, B., \& Pearl, D. K. (1979). Subjective referral of the timing for a conscious sensory experience: A functional role for the somatosensory specific projection system in man. Brain, 102, $191-222$.

Lotze, R. H. (1852). Medicinische Psychologie oder Physiologie, Seele. Leipzig: Weidmannsche Buchhandlung.

Lotze, M., Montoya, P., Erb, M., Hülsmann, E., Flor, H., Klose, U., Birbaumer, N., \& Grodd, W. (1999). Activation of cortical and cerebral motor areas during executed and imagined hand movements: An fMRI study. Journal of Cognitive Neuroscience, 11, 491-501.

Maturana, H. R., \& Varela, F. J. (1992). The tree of knowledge: The biological roots of human understanding. Boston: Shambhala Publication.

McFarland, D., \& Bösser, T. (1993). Intelligent behavior in animals and robots. Cambridge, MA: MIT Press.

Maes, P. (1991). A bottom-up mechanism for behavior selection in an artificial creature. In J. A. Meyer \& S. W. Wilson (Eds.), From animals to animats (pp. 238-246). Cambridge, MA: MIT Press.

Melzack, R. (1990). Phantom limbs and the concept of a neuromatrix. Trends in Neurosciences, 13, 88-92.

Metzinger, T. (2000). The subjectivity of subjective experience: A representationalist analysis of the first-person perspective. In T. Metzinger (Ed.), Neural correlates of consciousness: Empirical and conceptual questions. Cambridge, MA: MIT Press.

Morasso, P. G., Baratto, L., Capra, R., \& Spada, G. (1999). Internal models in the control of posture. Neural Networks, 12, 1173-1180.

Mussa Ivaldi, F. A., Morasso, P., \& Zaccaria, R. (1988). Kinematic networks-A distributed model for representing and regularizing motor redundancy. Biological Cybernetics, 60, 1-16.

Nilsson, N. J. (1998). Artificial intelligence. A new synthesis. San Francisco, CA: Morgan Kaufman Publishers Inc.

O’Regan, J. K., \& Noë, A. (2002). The origin of "feel”. In B. Hallam, D. Floreano, J. Hallam, G. Hayes, \& J.-A. Meyer (Eds.), From animals to animats (Vol. 7, pp. 27-35). Cambridge, MA: MIT Press.

Parisi, D., \& Cecconi, F. (1995). Learning in the active mode. In F. Moran, A. Moreno, J. J. Merelo, \& P. Chacón (Eds.), Advances in artificial life. Proceedings of the 3rd European Conference on Artificial Life (pp. 439-462). Berlin: Springer.

Pfeifer, R. (1995). Cognition-Perspectives from autonomous agents. Robotics and Autonomous Systems, 15, 4769.

Pollack, J. (1991). The induction of dynamical recognizers. Machine Learning, 7, 227-252.

Povinelli, D. J., Landry, A. M., Theall, L. A., Clark, B. R., \& Castille, C. M. (1999). Development of young children's understanding that the recent past is causally bound to the present. Developmental Psychology, 35, $1426-1439$.

Prinz, W. (1997). Why Donders has led us astray. Theoretical issues in stimulus-response compatibility. Advances in Psychology, 118, 247-267.

Ramachandran, V. S., \& Blakeslee, S. (1998). Phantoms in the brain. Probing the mysteries of the human mind. New York: Morrow.

Ramachandran, V. S., Dogers-Ramachandran, D., \& Cobb, S. (1995). Touching the phantom limb. Nature, 377, 489-490.

Rizzolatti, G., \& Arbib, M. A. (1998). Language within our grasp. Trends in Neurosciences, 21, 188-194.

Shepard, R. N., \& Metzler, J. (1971). Mental rotation of three-dimensional objects. Science, 171, 701-703.

Sakata, H., Taira, M., Kusunoki, M., Murata, A., \& Tanaka, Y. (1997). The parietal association cortex in depth perception and visual control of hand action. Trends in Neurosciences, 20, 350-357.

Sloman, A. (2000). Architectural requirements for human-like agents both natural and artificial. (What sorts of machines can love?). In K. Dautenhahn (Ed.), Human cognition and social agent technology (pp. 163-195). Amsterdam: John Benjamins.

Smith, M. A., \& Shadmehr, R. (2000). Error correction and the basal ganglia. Trends in Cognitive Sciences, 4, 367-369.

Steinkühler, U., \& Cruse, H. (1998). A holistic model for an internal representation to control the movement of a manipulator with redundant degrees of freedom. Biological Cybernetics, 79, 457-466.

Tani, J. (1998). An interpretation of the "self" from the dynamical systems perspective: A constructivist approach. Journal of Consciousness Studies, 5, 516-542. 
Tani, J., \& Nolfi, S. (1999). Learning to perceive the world as articulated: An approach for hierarchical learning in sensory-motor systems. Neural Networks, 12, 1131-1141.

Tinbergen, N. (1951). The study of instinct. London: Oxford University Press.

Ullman, S. (1995). High-level vision: Object recognition and visual cognition. Cambridge, MA: MIT Press.

Uszkoreit, H. (2000, Spring). Talk given at the meeting "Autonomie und Adaptivität", Kloster Seeon.

Vogeley, K., Bussfeld, P., Newen, A., Herrmann, S., Happé, F., Falkai, P., Maier, W., Shah, N. J., Fink, G. R., \& Zilles, K. (2001). Mind reading: Neural mechanisms of theory of mind and self-perspective. NeuroImage, 14, 170-181.

Walter, J., \& Ritter, H. (1996). Rapid learning with parametrized self-organizing maps. Neurocomputing, 12, 131153.

Weizsäcker, V. (1950). Der Gestaltkreis. Stuttgart: Thieme. 